Journal of Environmental Science and Public Health

doi: $10.26502 /$ jesph.96120029

Volume 2, Issue 1

Review Article

\title{
An Exploration into Wind Turbines, Their Impacts and Potential Solutions
}

\author{
Hiwa Mohammad Qadr*
}

Department of Physics, College of Science, Raparin University, Sulaimanyah, Iraqi Kurdistan, Iraq

*Corresponding Author: Hiwa Mohammad Qadr, Department of Physics, College of Science, Raparin University, Sulaimanyah, Iraqi Kurdistan, Iraq, E-mail: hiwa.physics@uor.edu.krd

Received: 08 February 2018; Accepted: 22 February 2018; Published: 28 February 2018

\begin{abstract}
This review considers some environmental problems and solutions associated with turbines and their impact on humans and birds. The objective is to explore the relationship between noise pollution, electromagnetic interference and visual impact problems that how wind farm create stress for nearby residents and will refer to problems of avian collision with wind turbines. Furthermore, it will be shown that it includes result of solutions for reducing these problems.
\end{abstract}

Keywords: Noise pollution; Avian collision; Disturbed sleep; Visual impact; Birdlife

\section{Introduction}

Over the past few years, we have been able to see noticeably higher costs for electricity needs. Electricity prices have been climbing rapidly because the number of people in the world has increased, along with machines they buy for their homes that consume electricity. There has been a dramatic rise in capacity for electricity generation from wind energy. Therefore, the experts have developed wind power as a renewable energy resource that is constantly recycled in nature. Nevertheless, despite all the work in this area, it seems that, as well as benefits, wind power can also have disadvantages for humans and wildlife.

Environmental impacts associated with wind energy can be both positive and negative. On the positive side, wind turbines can be more environmentally friendly than other fuels. Other sources of energy, such as fossil fuels and nuclear power, are particularly known to produce pollution. On the negative side, wind turbines can create problems 
for people who are living in the vicinity, such as disturbed sleep and stress because of noise, visual impact and electromagnetic interference [1]. Additionally, according to [2] "some negative environmental aspects have been shown to be especially important in populated or scenic areas."

Accordingly, this review will explain wind energy and demonstrate the complex relationship between wind turbines and humans and nature, specifically birds. Furthermore, it will be shown that it includes result of solutions for reducing these problems.

\subsection{Stress effect and wind turbines}

Stress has been defined as reaction with human's body to a change, which requires a mental, physical or responses to the stressors. It has been demonstrated that stressors can be having the difference between people that located on a person compared with other people. There is no doubt that wind turbines have developed swiftly in the world and there appear to be some drawbacks, which may cause stress or suffering to people in the vicinity, especially with large wind farms. This can unfortunately result in mental health and sleep problems, when wind turbines produce a combination of noise pollution and electromagnetic interruptions. Furthermore, increased stress can damage the human brain and cause anxiety, anger and high blood pressure.

\section{Problems}

\subsection{Noise pollution caused by wind turbines}

Noise pollution problems, associated with stress, have undoubtedly been particularly studied in respect of environmental issues and wind energy. Noise levels are usually measured in a unit that is called decibel (dB), weighted by the sensitivity of the human ear. In contrast, loud music might be heavy that may be relaxing and desirable for people. Normally, most of the human population can hear sounds which have frequencies in-between approximately $20 \mathrm{~Hz}$ infrasound to $20000 \mathrm{~Hz}$ ultrasound. As result of this, not all of us can hear sounds with frequency outside of this range [3]. Some frequencies cause unpleasant emotions for humans, as with some wind turbines (like unwanted sounds such as a car horn and an alarm clock); different levels of frequency, intensity, and frequency distribution affect concerns about noise [2].

It has been claimed by WHO cited in [3] that environmental noise can mainly have different mental impacts due to machines as wind turbines, for instance emotional stress, headache, anxiousness and social conflicts. Plenty of wind turbines have been put into rural areas where there is a lower population, as this reduces the impact and stress caused [2].

Furthermore, the noise pollution produced by wind turbines can disturb sleep in various ways. For example, people living near to the wind farms, maybe awake overnight, difficulty to sleep very well and felling very tired in the morning. This resultant sleep disturbance leads to biological effects, for instance the level of stress hormone change, 
unwanted movement of the people during sleep. Such effects may not be very noticeable at first, but do affect health in the long term [3].

Despite the increase in the number of wind turbines caused by increasing in the rate of heart beat and blood pressure due to massive noise. It is clear that it is important to explore solutions to decrease the problem.

\subsection{Visual effects caused by wind turbines}

It seems that constructing new artificial wind farms in the countryside land can have an extraordinary consequence on these regions and make anxiety for their residents. In fact, the components of nature are likely to be organized appealingly in a balance condition. It is probable that the attractiveness of the environment and countryside may be devastated where wind turbines have been built in these areas. One of wind power's observed difficult environmental impact issues, as well as a major important matter from the general public, is visibility. Although, when compared with other environmental impact related to wind power, the visual impact could be the least serious [2].

For instance, the optical impacts and countryside observations have been considered as key natural problems in establishing the applications of wind farm related to wind energy improvement as visual impacts and landscape are by nature subjective and altering over period and place. The countryside's and optical effects such as features like substation constructions, grid connection, access and place tracks, turbines (size, height, number, material and colour), communication lines and anemometer masts [4].

According to [5], wind turbines have an extremely noticeable structure in any countryside due to their high capacity. In general, the people who are resident near wind turbines are more affected compared to those citizens who live far away. In contrast, many people believe that this can be changed when the huge numbers of turbines lead to breaking the optic impact of the countryside, as people will simply be used to them. For example, larger turbines can be more suitable than small turbines. Additionally, the larger turbines don't appear as noticeable to human eyes, because their rotational speed is slower than small turbines.

On the other hand, [6] disagree that turbines have an attractive beautification, and they can appeal to travellers, encouraging them to visit those areas containing some wind turbines. Generally, the people most anxious about the environment are the ones who are likely to be the most stressed by its visual impact. Thus, this issue can mainly be solved by reducing stressful implications on the population.

\subsection{Electromagnetic interference effects of wind turbines}

Wind turbines create electromagnetic interference (EMI) problems involving X-Rays and ultra violet rays. EMI can be defined as disturbance that affects an electrical field. Typically, any kind of big structure, whether moving or stationary, stationary, might degrade and interfere with the transmission of electromagnetic signals. It is likely that 
their vertical structure can reflect portions of the electromagnetic radiation in a way that interferes with the original signal arriving at the receiver. Several people have suggested that wind turbines could lead to interference and impact on radio transmissions and TV when they rotate blades that can disrupt the signals [2].

In addition, effects of electromagnetic interference, including scattering of signals and blocking, might affect household TV and other electronic devices, which causes stress to people, additionally to any long-term health implications.

\subsection{Avian collisions with wind turbines}

Wind turbines can also affect birds and other flying animals such as bats, when they interact with rotor blades. As the result of this, they are being killed and injury by wind turbines because they fly around offshore or in rural areas. In general, there are two significant categories of impact. Firstly, it can directly impact, for example, via serious risks to birds and bats flying into the machines, due to the huge pressure around the turbines. Secondly, there is also indirect impact, including disturbance of breeding and staging, consequently affecting migration and travelling birds disturbing effect on migrating as well as travelling birds [2, 5].

It has been claimed by [1] when the turbine is blended with the skyline, this may cause more birds to be killed. However, according to a study by BUND in Germany cited in [5], "there is statistically one bird death per year for two turbines, or 8000 bird deaths per year. 5-10 million birds die in road traffic and the same number again in power lines".

In conclusion, wind turbines are not benefit and suitable for birds but it may not be a huge problem, and there are solutions to ease the problem

\section{Solutions}

\subsection{Solutions to the noise pollution of wind turbines}

Noise from wind turbines is generated by moving blades, but reduction of wind turbines noise has to occur by one of two main ways. Firstly, the moving components such as gearbox and generator produce mechanical noise. Secondly, aerodynamic noise is created by interaction of the blades of a turbine with wind [1]. Data that has been collected when wind farms are developed, suggest that modern wind turbines designs appear to reduce noise pollution when compared with older models [4]. According to [3] mechanical noise can be reduced power, which generated by wind turbines during mid-night and impacts on nearby residents therefore putting a long distance between wind farms and the occupants. Moreover, aerodynamic noise maybe decreases by limiting the number of cycle blades per a minute.

In summary, it is believed that noise pollution from wind turbines does create some problems, but it can be solved using some patterns of action, outlined above Furthermore, Impact on people reduces according to distance, so the fewer people close to the turbines, the better. 


\subsection{Solutions to the visual impact from wind turbines}

The second area for solutions, is regarding visual impact of turbines. Although many people believe that wind turbines look attractive, but a majority of the population consider wind turbines unattractive, so the main solution is to minimise their visual impact. According to [1], the equipment quality of wind energy generators can create a substantial contribution to this issue. Features that help to minimize visual impact include their colour, size of wind turbine, the number of blades and distance between the residences and wind turbines.

First of all, the colour of wind turbines should be designed using colours suitable for the reduction of the visual impact [5]. Secondly, the distance from dwellings has been recognized as a significant attributing factor in minimizing wind turbines pressure on people. From further away, the rotational blades appear lower, not attracting people's eyes when they are moving [1]. Therefore, ideally they need to be built high up to catch the most wind, but far away to lessen the impact.

\subsection{Solutions to the electromagnetic interference from wind turbines}

The third factor that can create stress is electromagnetic interference and there are some solutions to this. Modern blades are produced using synthetic materials, which when substituted for metal blades reduce interference to mobile and TV signals and have a lower effect on the electromagnetic radiation transmission. Electrical systems, which are maintained well, should not suffer too much interference. Overall, when wind turbines are sited close to residents, interference can prove very difficult to rule out [2].

\subsection{Solutions to the avian collisions from wind turbines}

Thinking about solutions to avian collisions, there are three potential ways to reduce the danger to flying creatures with turbines, and thus reduce the associated environmental impact. Firstly, an increase in the visibility of rotor blades. Secondly, use turbines that have white flashing lights, not red ones. Thirdly, protect migration paths when birds fly in large numbers. Using these three patterns, birds could be helped to avoid collision with wind turbines Thus, in three patters, birds may be avoiding interaction. Process of the world by wind turbines is causes of killing the average number of birds and other flying animals, but as can be seen from the above solutions that cause declining impacts on them.

\section{Conclusion}

This work has looked at the context and background to wind turbines, and some of the problem areas they can create, in terms of noise, visual impact, interference and the risk of collision in the bird population. Following this, some approaches to limit the negative impact and stresses they can produce, were outlined.

As this report has demonstrated, the environmental impacts of wind turbines can create significant stress for residents who are living the vicinity. Such stress could potentially lead to psychological distress and various physical health and well-being problems. It appears that these symptoms could be directly linked to pollution, visual impact 
and electromagnetic interference associated with wind turbines. The work has also outlined ways in which wind turbines can impact on birdlife.

It has been suggested that if wind turbines are planned and designed carefully, many of these negative impacts may be eliminated or reduced in terms of the amount of stress on people [1]. Ways to do this, which have been discussed, include altering colour, design, construction material, taking care over planning sites and selecting exact locations with care and attention to the potential problems. For example, there is still disagreement about whether wind turbines should be located onshore or offshore, and which location creates the most impact. There is no doubt that when wind turbines have been built far from inhabitants, an amount of electrical energy may be lost due to using electric resistance wire for transferring the generated electricity from turbines to houses. Therefore, inventors suggest the best solution to discover this problem.

Finally, despite the negative impacts, solutions are being found, as has been discussed, and it seems clear that wind turbines have a lot of potential to be a cleaner energy source, with lower impact than many other fuels, and may be part of a future global strategy to address climate change issues.

\section{References}

1. Saidur R, Rahim N, Islam M, et al. Renewable and sustainable energy reviews 15 (2011): 2423-2430.

2. Manwell JF, McGowan JG, Rogers AL. Wind energy explained: theory design and application John Wiley and Sons (2010).

3. Bowdler D, Leventhall G, Raspet R. Journal of the Acoustical Society of America 132 (2012): 1233.

4. Association E.W.E. Wind energy-the facts: a guide to the technology economics and future of wind power Routledge (2012).

5. Energy G. R. http://www.greenrhinoenergy.com/renewable/wind/wind_environment.php. 2013.

6. Müller DK, Jansson B. Tourism in peripheries: perspectives from the far north and south Cabi (2006).

Citation: Hiwa Mohammad Qadr. An Exploration into Wind Turbines, their Impacts and Potential Solutions. Journal of Environmental Science and Public Health 2 (2018): 64-69.

(C)
Creative Commons Attribution (CC-BY) license 4.0 